\title{
Actinotignum Schaalii bacteremia: from microbiota to the infection
}

\author{
Sara Pinto. María Figueiredo, Fatima Leal-Seabra, Catarina Pereira \\ Centro Hospitalar Vila Nova de Gaia Espinho
}

\begin{abstract}
Actinotignum schaalii is a gram-positive, facultative anaerobic bacillus, an opportunist agent that is underestimated or mistaken as a contaminant.

In this article, we present a case of sepsis with multiorgan dysfunction due to acute pyelonephritis complicated by $A$. schaalii bacteraemia in an elderly patient with diabetes mellitus, chronic obstructive renal disease, benign prostatic hyperplasia, renal lithiasis and chronic urinary device. Through specific laboratory techniques such as Matrix-Assisted Laser Desorption/lonisation Time-Of-Flight Mass Spectrometry, it was possible to identify the presence of A. schaalii in blood cultures. However, uroculture was negative. After 20 days of $\bigotimes$-lactam, it was possible to successfully heal the infection.

The difficulty in identifying this rare agent has definitely constrained our diagnostic and therapeutic approach.

Keywords: Actinotignum Schaalii, bacteremia, urinary tract infection.
\end{abstract}

Palabras clave: Actinotignum Schaalii. BActeriemia. Infección del tracto urinario.

\section{Introduction}

Actinotignum schaalii is a facultative anaerobic gram-positive bacillus with slow growth in blood-enriched medium in anaerobic or under $5 \%$ carbon dioxide environment ${ }^{1,2}$. The reservoir of this bacterium is unknown. It was found in genitourinary tract, skin and vaginal mucosa ${ }^{1}$ and it is responsible for urinary infections (UTI) in elderly². These characteristics are responsible for underdiagnosed and unreported infections by this microorganism ${ }^{2-4}$.

The authors describe a case of sepsis by Actinotignum schaalii, whose clinical, evolution and antimicrobial strategy require reflection.

\section{Clinical case}

An 80-years old man was found in the emergency department presenting with hypogastric pain and fever for the past 24 hours. In addition, there has been a recent history of benign prostatic hyperplasia with acute urinary retention and bladder catheter since the previous month. He presented the following comorbidities: type 2 diabetes mellitus treated with metformin, obesity, dyslipidaemia, chronic renal disease, atrial fibrillation and peptic ulcer disease.

In the emergency department, his temperature was $38.2^{\circ} \mathrm{C}$, blood pressure $(102 / 58 \mathrm{mmHg}$, heart rate 90 beats/min, without respiratory distress. His abdomen was soft and painless. Laboratory tests showed a white-cell count of 9860 per cubic millimeter (normal range, 4000 to 11,000), a platelet count of 72,000 per cubic millimeter (normal range, 150000 to 400000) and C-reactive protein of $30,6 \mathrm{mg} / \mathrm{dL}$. The serum creatinine level was of 3,2mg per deciliter (reference range, 0,6 to 0,9 $\mathrm{mg}$ per deciliter; the creatinine level had been $1,2 \mathrm{mg}$ per deciliter 3 months earlier) with diuresis of of $0,26 \mathrm{mililiter/Kilogram/}$ hour on first 24 hours. The arterial blood gas showed pH 7.41 (normal range, 7,35-7,45), Pa02 69mmHg (normal range, 80$100 \mathrm{mmHg}$ ), PaCO2 26mmol/L (normal range, $36-40 \mathrm{mmHg}$ ), HCO3- 17 (normal range, 22-26 mEq/L), base excess of -6,5 (normal range, -2 to $+2 \mathrm{mmol} / \mathrm{L}$ ), lactate level of 8,2 (normal range, $0,5-2,2 \mathrm{mmol} / \mathrm{L})$. In the urinalysis, white-blood cells was $>10$ per high power field (normal range inferior to 5 per high power field) and erythrocytes 5-10 per high power field (normal range, inferior to 0,5 per high power field), without nitrites. The bladder, kidney and prostatic ultrasound showed right hydroureteronephrosis $(21 \mathrm{~mm})$ without the identification of the position and cause of the obstruction and benign prostatic hypertrophy. Urological tomography confirmed a presence of a 3-4 mm calcification located in the urethral meatus with urolithiasis in the right and lower right calyx groups.

The patient was hospitalized due to urosepsis with multiple organ failure. Blood and urine cultures were collected. A treatment with ceftriaxone was empirically started. Anaerobic blood culture revealed a gram-positive bacillus of 24 hours incubation with small and grey colonies on blood-enriched microbiological culture media. Vitek2 $B$ identification card showed an anaerobic and coryneform bacteria, confirmed to be Actinotignum schaalii, by MALDITOF MS (Matrix-Assisted Laser Desorption / Ionisation Time-Of-Flight Mass Spectrometry). The urine culture was negative. After 72 hours of hospitalization, the patient maintained persistent fever, hypotension (which means that the arterial pressures were $60 \mathrm{mmHg}$ ), and oliguric acute kidney failure. New blood and urine cultures were collected. Another infection and complications were excluded. The treatment was changed from ceftriaxone to piperacillin/tazobactam. Finally, it was possible to identify the Actinotignum schaalii in the first blood cultures. The patient's treatment was changed another time again from piperacillin/tazobactam to ceftriaxone, to complete the 20 days of therapy. After this change, the patient had clinical improvement as he had no fever and hemodynamic stability, lower inflammatory parameters and resolution of acute kidney dysfunction. A second set of blood cultures was negative. Six months later, he remained with no fever or urinary reinfection. 


\section{Discussion}

Actinotignum schaalii is a gram-positive, facultative anaerobic, catalase-negative bacterium with slow growth in bloodenriched medium in an anaerobic environment or under $5 \%$ carbon dioxide, often associated with the simultaneous growth of other bacteria ${ }^{1-3,5}$. These conditions are associated with the underdevelopment of this agent in the culture media, so its prevalence in the population may be underestimated ${ }^{2-4}$.

Some studies in healthy populations suggest that Actinotignum schaalii may be a commensal of the urogenital flora, distinguishing itself from the most common uropathogens because it does not originate from the gastro-intestinal tract $2,6,7$.

UTI by Actinotignum schaalii are more common in the elderly, with some studies showing male predominance (3). It was associated with predisposing conditions such as chronic urinary devices, benign prostatic hypertrophy, chronic renal disease, immunosuppression, prostatic or bladder cancer, urinary incontinence, neurogenic bladder and urethral stricture ${ }^{2,3,7}$. This patient had several of these risk factors: age, gender and clinical history of, diabetes, chronic kidney and prostate diseases.

Although this agent is associated with UTI7, in a retrospective study of cases of bacteraemia by Actinotignum schaalii, only one patient had isolation of this agent in the urine culture, despite the 36 cases in which the primary focus was assumed to be urinary based on clinical and predisposing factors ${ }^{3}$. In this case, UTI was diagnosed based on clinical picture, but the urine culture was negative, similar to other cases described in the literature ${ }^{3,8,9}$. Laboratory techniques are now available with better sensitivity for these rare and specific agents, such as MALDITOF MS or 16S rRNA sequencing. MALDITOF appears to be a more accurate and fast method of identifying Actinotignum schaalii than $16 \mathrm{~S}$ rRNA sequencing ${ }^{2-4,7}$. Unfortunately, these techniques are not always available and can lead to delays in the identification of this bacterium, as it was verified in the presented case-

The clinical picture of infections by Actinotignum schaaliiis is similar to other UTI. In the case described, clinical worsening after $72 \mathrm{~h}$ of ceftriaxone treatment justified the adjustment of therapy to piperacillin/tazobactam, because the agent was still unknown until that moment. There was no recurrence of fever or organ dysfunction after the adjustment of antibiotic therapy to ceftriaxone, showing its efficiency in controlling the infection.

Actinotignum schaalii appears to be susceptible to all Q-lactams and with low minimum inhibitory concentrations $(<0.5 \mathrm{mg} / \mathrm{L})^{2,5,8,9}$. Nevertheless, there was an intrinsic resistance to several antimicrobials ${ }^{2,9}$, such as trimethoprim and quinolones $2,3,5,8,9$, which may be related to the production of fimbriae. There haven't been any recommendations up to now on antimicrobial susceptibility testing for this genus or ranges for evaluation of minimum inhibitory concentration ${ }^{2}$. The length of the treatment is still uncertain, but there are reports of recurrence after one week of treatment. So, taking into account the site of infection ${ }^{2,8}$, it is suggested, at least, two weeks of treatment. In the described case, we chose to extend the antibiotic therapy due to the delay in clinical resolution, which revealed to be a successful strategy, because there was no recurrence of the infection.

Mortality from bacteraemia by Actinotignum schaalii is high. Despite the presence urosepsis with multiorgan dysfunction, the evolution of the clinical case was positive successful, without any sequelae and remained asymptomatic after a 6-month follow-up.

\section{Conclusion}

Actinotignum schaalii is an uropathogen associated with bacteraemia. The identification of this agent is essential because it is intrinsically resistant to several antibiotics used empirically in the treatment of UTI and requires long-lasting courses of antibiotherapy. Therefore, the use of the latest laboratory techniques for the identification of microorganisms, such as MALDITOF MS, is clearly critical in these clinical cases.

\section{Acknowledgments}

The authors acknowledge Centro Hospitalar Vila Nova Gaia/Espinho - Laboratório de Microbiologia assistance in this case, specifically Dra Gabriela Abreu, for their assistance in this case. They also thank Dr. Agripino Oliveira, for further assistance and aiding in our collaboration.

\section{References}

1. Lotte R, Lotte L, Ruimy R, et al. Actinotignum schaalii (formerly Actinobaculum schaalii): a newly recognized pathogen-review of the literature. Clin Microbiol Infect. 2016; 22: 28-36.

2. Yassin AF, Spröer C, Pukall R, Sylvester M, Siering C, Schumann P. Dissection of the genus Actinobaculum: Reclassification of Actinobaculum schaalii Lawson et al. 1997 and Actinobaculum urinale Hall et al. 2003 as Actinotignum schaalii gen. nov.,. comb.. nov. and Actinotignum urinale comb. nov., description of Actinotignum sanguinis sp. nov. and emended descriptions of the genus Actinobaculum and Actinobaculum suis; and re-examination of the culture deposited as Actinobaculum massiliense CCUG 47753. Vols. 5DSM 19118T), revealing that it does not represent a strain of this species. International Journal of Systematic and Evolutionary Microbiology. 2015; 65, 615-624

3. Pedersen $\mathrm{H}$, Senneby E, Rasmussen M. Clinical and microbiological features of Actinotignum bacteremia: a retrospective observational study of 57 cases. Eur $\mathrm{J}$ Clin Microbiol Infect Dis. 2017 e 36:791-796.

4. Stevens RP, Taylor PC. Actinotignum (formerly Actinobaculum) schaalii: a review of MALDI-TOF for identification of clinical isolates, and a proposed method for presumptive phenotypic identification. Pathology. 2016 e 48(4).

5. Nielsen HL, Søby KM, Christensen JJ, Prag J. Actinobaculum schaalii: A common cause of urinary tract infection in the elderly population. Bacteriological and clinical characteristics. Scandinavian Journal of Infectious Diseases, 2010 e 43-47., 42.

6. Olsen AB, Andersen PK, Bank S, Søby KM, Lund L, Jørgen Prag J. Actinobaculum schaalii, a commensal of the urogenital area. BJU International. 2013 e 112 , 394-397.

7. Prigent G, Perillaud C, Amara M, Coutard A, Blanc C, Pangon B. Actinobaculum schaalii: A truly emerging pathogen?. New Microbe and New Infect 2016 e 8-16.

8. Kristiansen R, Dueholm MS, Bank S, Nielsen PH, Karst SM, Cattoir V, et al. Complete Genome Sequence of Actinobaculum schaalii Strain CCUG 27420. Genome Announc. 2014 e 2(5):00880-14.

9. Reinhard M, Prag J, Kemp M, Andresen K, Klemmensen B, Højlyng N, et al. Ten Cases of Actinobaculum schaalii Infection: Clinical Relevance, Bacterial Identification, and Antibiotic Susceptibility. J. Clin. Microbiol. 2005 e 43 (10), 5305-5308. 